\title{
Efficient Oxidative Desulfurization of Model Oil at Room Temperature with Ionic Liquid as Extraction Solvent
}

\author{
Harold Henrison C. Chiu ${ }^{a}$, Susan D. Arco ${ }^{b, *}$, Zhang Chun Ping ${ }^{c}$, and \\ Nelson R. Villarante ${ }^{a, *}$
}

${ }^{a}$ Department of Physical Sciences and Mathematics, College of Arts and Sciences, University of the Philippines Manila, Ermita, Manila, Philippines

${ }^{b}$ Institute of Chemistry, College of Science, University of the Philippines, Diliman, Quezon City, Philippines

'Division of Biotechnology and Pharmaceuticals, Keyan Road, Zhunan Town, Miaoli County, Taiwan, R.O.C.

The oxidative desulfurization of model oil (hexane solution of thiophene) was carried out at room temperature in a two-step method involving: 1) the acetic acid catalyzed oxidation of thiophene with hydrogen peroxide and 2) the subsequent extraction of the oxidized products with three 1-alkyl-3-methylimidazolium bromide [RMIM]Br ionic liquids of varying alkyl substituent $\mathrm{R}$ chain length $\left(\mathrm{R}: \mathrm{C}_{2}, \mathrm{C}_{4}, \mathrm{C}_{6}\right)$ and with acetonitrile as control. For purposes of comparison, a non-oxidative extractive desulfurization of model oil with the above ionic liquid and with acetonitrile was also performed. The thiophene extraction efficiencies of the ionic liquids and that of the control in both the oxidative and non-oxidative procedures were determined by means of gas chromatography. The ionic liquid of the shortest alkyl substituent chain length $\left(\mathrm{R}: \mathrm{C}_{2}\right)$, [EMIM] Br exhibited the highest extraction efficiency in the oxidative desulfurization of the model oil; the extraction efficiency of [EMIM] $\mathrm{Br}$ was also observed to exceed that of acetonitrile. In general, the oxidative desulfurization with the above [RMIM]Br's is apparently a more efficient method of thiophene removal from the model oil as compared to a non-oxidative procedure with the same extraction solvents. The extraction efficiency of [RMIM]Br's was observed to decrease with the lengthening of the alkyl substituent chain. The same trend is observed in the non-oxidative extractive desulfurization of the model oil. Recyclability analysis of [EMIM] $\mathrm{Br}$ showed that $[\mathrm{EMIM}] \mathrm{Br}$ can be recycled thrice with no significant decrease in extraction efficiency.

Keywords: ionic liquids; desulfurization; liquid-liquid extraction; [EMIM] [Br]; thiophene

\section{INTRODUCTION}

Sulfur compounds in the SOx emissions from the combustion of fuels are among the primary sources of air pollution and are a major source of acid rain (Zhao et al., Optimization, 2010). These compounds are also the leading cause of many serious human

\footnotetext{
*Author to whom correspondence should be addressed; email: susan_arco@yahoo.com.ph,nrv41@yahoo.com
} 
respiratory system diseases such as lung cancer (Zhao et al., Oxidation, 2009). The sulfur limit for fuel cell (both for automotive and stationary systems) liquid fuels is being gradually decreased and an S-content of less than $10 \mathrm{ppm}(\mathrm{w} / \mathrm{w}) \mathrm{S}$ is desirable (Seeberger and Jess, 2009). Fuel sulfur content removal process operation based on catalytic hydrodesulfurization (HDS), a traditional desulfurization method (Zhao et al., Optimization, 2010; Seeberger and Jess, 2009) proves to be tedious and costly in that it requires high pressure and high temperature, as well as the use of catalysts such as CoMo or NiMo. Furthermore, while HDS proves to be efficient for the removal of aliphatic sulfur compounds, HDS efficiency in the removal of aromatic sulfur compounds such as benzothiophene and dibenzothiophene is limited. Among the alternative methods of desulfurization, extractive desulfurization is viewed to be among the most efficient but its industrial scale application entails the use of large volumes of volatile organic compound (VOC) extractants (Zhao et al., Optimization, 2010). To alleviate this problem, the extraction of sulfur compounds from fuels through ionic liquids (ILs) has been studied extensively (Likhanova et al., 2010). ILs are non-volatile, easy to handle, thermally robust, and non-explosive "designer solvents". Being designer solvents, their physical and chemical properties can be altered to suit an application of interest through modifications on their cation and anion component (Bosmann et al., 2001; Cheng et al., 2008). Lewis and Brønsted -acidic ionic liquids employing mixtures of $n$ butyl-3-methylimiazolium chloride and $\mathrm{n}$ ethyl-3-methylimidazolium chloride with $\mathrm{AlCl}_{3}$ have been proven effective for the extraction of sulfur containing compounds (Likhanova et al., 2010; Francisco et al., 2010) but these mixtures are unstable in air and sensitive to water making these of limited practical utility (Francisco et al., 2010).

Another alternative desulfurization process which requires moderate conditions and does not involve the use of catalysts is oxidative desulfurization (ODS). ODS is a two-step process (Lo et al., 2003) involving the oxidation of sulfur compounds in fuels to sulfones and the subsequent extraction of these sulfones with polar solvents. However, an industrial scale application of ODS, as with HDS also involves the use of VOC extractants (Zhao et al., Optimization, 2010; Lo, 2003). In response to this, Lo (2003) has investigated on the removal of sulfurcontaining compounds from light oils through ODS by a combination of both chemical oxidation and solvent extraction using the IL 1-butyl-3-methylimidazolium hexafluorophosphate [BMIM] $\left.\mathrm{PF}_{6}\right]$ and 1-butyl-3methylimidazolium tetrafluoroborate [BMIM] $\left[\mathrm{BF}_{4}\right]$. This approach to ODS is proven to improve the yield of desulfurization by roughly one magnitude (Lo et al., 2003) relative to that with extraction using ILs alone. A further advantage of Lo's approach is that the ionic liquids can be recycled after workup. In more recent studies, a fluoride-free Bronsted acid IL [Hnmp] $\mathrm{H}_{2} \mathrm{PO}_{4}$ (Zhao et al., Oxidation, 2009) and several pyridinium based ionic liquids (Zhao et al., Oxidation, 2010) were utilized both as catalyst and extractant in a combined oxidative and extractive desulfurization of fuels. The use of [RMIM][Halide] ionic liquids in oxidative desulfurization holds the advantage of convenience in preparation because, unlike in the preparation of the previously mentioned IL's, this only involves a one-step, one-pot, thermal or ultrasoundassisted synthesis (Seeberger and Jess, 2009; Likhanova et al., 2010).

The typical procedure in a combined oxidative and extractive desulfurization of fuels involves mixing the fuel sample with the ionic liquid in a 1:1 volume-to-volume ratio (Jiang et al., 2008; Alonso et al., 2008). In experiments involving petroleum and fossil fuel products, model oils that simulate fossil fuels are often used in the optimization of protocols. These model oils should have components similar in structure or composition to fossil fuels. Their preparation includes dissolving small amounts of thiophene or its derivatives (aromatic sulfur compounds in fossil fuel) in suitable and readily available hydrocarbon solvents such as hexane, heptane, octane or tetradecane (Francisco et al., 2010; Lo et al., 2003). 
Hydrogen peroxide (oxidant) and acetic acid are then added to the fuel-IL mixture after which the mixture is stirred for a few minutes and equilibrated for 6 hours to 24 hours at temperatures of $25^{\circ} \mathrm{C}$ to $60^{\circ} \mathrm{C}$ (Lo et al., 2003).

In this study, we employed a combination of: 1) chemical oxidation of thiophene, and 2) extraction of its oxidized products with ionic liquids (Figure 1), in the desulfurization of model oil. Hexane solutions of thiophene were used as model oil (Francisco et al., 2010).<smiles>c1ccsc1</smiles><smiles>CC(C)(C)OO</smiles><smiles>O=S1(=O)C=CC=C1</smiles>

Figure 1. Chemical oxidation of thiophene into sulfone.

The ionic liquids (Figure 2), 1-ethyl-3methylimidazolium bromide [EMIM][Br], 1butyl-3-methylimidazolium bromide [BMIM] $[\mathrm{Br}]$, and 1-hexyl-3-methylimidazolium bromide [HMIM] $[\mathrm{Br}]$, which are immiscible with the model oil, were used as solvents for the liquid-liquid extraction.

Both the efficiency in desulfurization and recyclability of these ionic liquids were examined.

\section{METHODOLOGY}

Desulfurization. Oxidative desulfurization of the model oil was carried out as follows: $5 \mathrm{~mL}$ of the thiophene standard (1000 ppm S) was

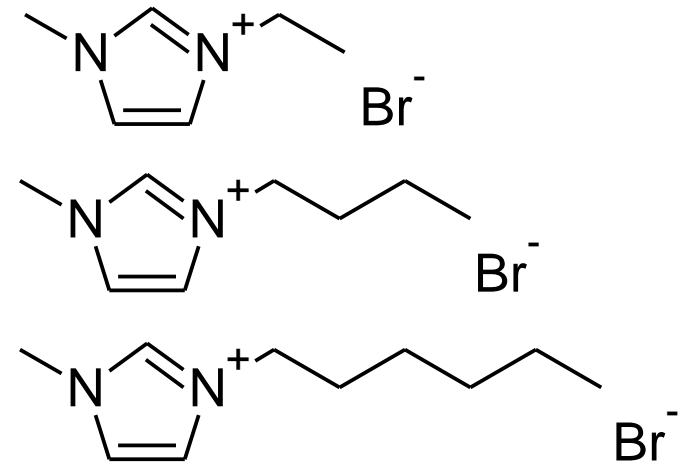

Figure 2. Structures of the ILs used in this study.

added to $5 \mathrm{~mL}$ of ionic liquid, and mixed with a vortex mixer for 1 minute. Hydrogen peroxide $(30 \%, 1.36 \mathrm{~mL})$ and glacial acetic acid $(0.36 \mathrm{~mL})$ were added to the above mixture and these were also mixed with a vortex mixer for 1 minute. The resulting mixture was staged for 24 hours for equilibration. Decrease in thiophene concentration in the hydrocarbon layer of this mixture was determined by gas chromatography with a Hewlett Packard P 5890 Series II GC operated under the conditions summarized in Table 1 . The above procedure applies in the non-oxidative desulfurization of the model oil but the addition of hydrogen peroxide and glacial acetic acid is excluded.

Regeneration of the Ionic Liquids. The ionic liquid was separated from the model oil by decantation. Residual water and oxidizing agents were removed through evaporation at $100^{\circ} \mathrm{C}$ for $3 \mathrm{~h}$ in a vacuum oven (Lo et al., 2003). The ionic liquid was then recovered from the residue by extracting it three times

Table 1. Chromatographic conditions for thiophene analysis.

\begin{tabular}{ll}
\hline Column & HP-FFAP Polyethylene Glycol TPA $(25 \mathrm{~m}$ x $200 \mu \mathrm{m} \times 0.30 \mu \mathrm{m}$ \\
Detector type & FID \\
Detector temp & $250^{\circ} \mathrm{C}$ \\
Carrier gas & Nitrogen \\
Injector temp & $250^{\circ} \mathrm{C}$ \\
Flow Rate & $1.5 \mathrm{~mL} / \mathrm{min}$ \\
Column oven & $n$-hexane: $70^{\circ} \mathrm{C}(1 \mathrm{~min}) \rightarrow 100^{\circ} \mathrm{C}\left(25^{\circ} \mathrm{C} / \mathrm{min}\right), 1 \mathrm{~min}$ \\
\hline
\end{tabular}



with $45 \mathrm{~mL}$ portions of ethyl acetate. The purity of the recovered ionic liquids was assessed by ${ }^{1} \mathrm{H}$ NMR (Varian Mercury-400, 1D Single Pulsed) and ${ }^{13} \mathrm{C}$ NMR (Varian Mercury-400, 1D Single Pulsed, Proton Decoupled) spectroscopy.

\section{RESULTS AND DISCUSSION}

Three imidazolium-based ionic liquids (IL) of varying alkyl substituent chain length, [EMIM][Br], [BMIM][Br], and [HMIM][Br] were used as extraction solvents in the oxidative desulfurization of model oil (Fu et al., 2006), hexane solution of thiophene. For purposes of comparison, a non-oxidative desulfurization of the model oil was also performed. The extraction efficiencies of the above ionic liquids were evaluated relative to acetonitrile- a solvent which is typically used in desulfurization (Kim et al., 2004).

It has been observed in both oxidative and non-oxidative desulfurization of [RMIM] $[\mathrm{Br}]$ ILs that [EMIM][Br], the ionic liquid of the shortest alkyl substituent, exhibits the highest extraction efficiency. The percentage removal (Figure 3) of sulfur by [EMIM][Br] are 47.03 $\pm 1.29 \%$ and $94.34 \pm 0.20 \%$ for the nonoxidative and oxidative desulfurization of model oil, respectively. It is apparent that the extraction efficiency increases two-fold when thiophene is first oxidized into sulfoxide and sulfone. This trend is in agreement with previous studies (Lo et al., 2003; Kim et al., 2012) wherein the oxidation of thiophene and its derivatives leads to a more efficient extraction of the oxidized products (sulfoxides and sulfones of thiophene) into the ionic liquid phase. This increase in efficiency can be attributed to a better complementarity of the polarity of the ionic liquids with those of the sulfoxides and sulfones (Lo et al., 2003; Kim et al., 2004; Chen et al., 2006; Dekhordi et al., 2009) relative to that with unoxidized thiophene.

The extraction efficiency of the three ionic liquids has been observed to decrease with the increase of alkyl substituent chain length from 2 carbons in [EMIM] $\mathrm{Br}$ ] to 6 carbons in [HMIM] $[\mathrm{Br}]$; [BMIM] $[\mathrm{Br}]$, the ionic liquid of intermediate chain length, exhibits an extraction efficiency which is comparable with that of acetonitrile. Studies (Lu et al., 2007; Ahosseini and Scurto, 2008; Gardas and

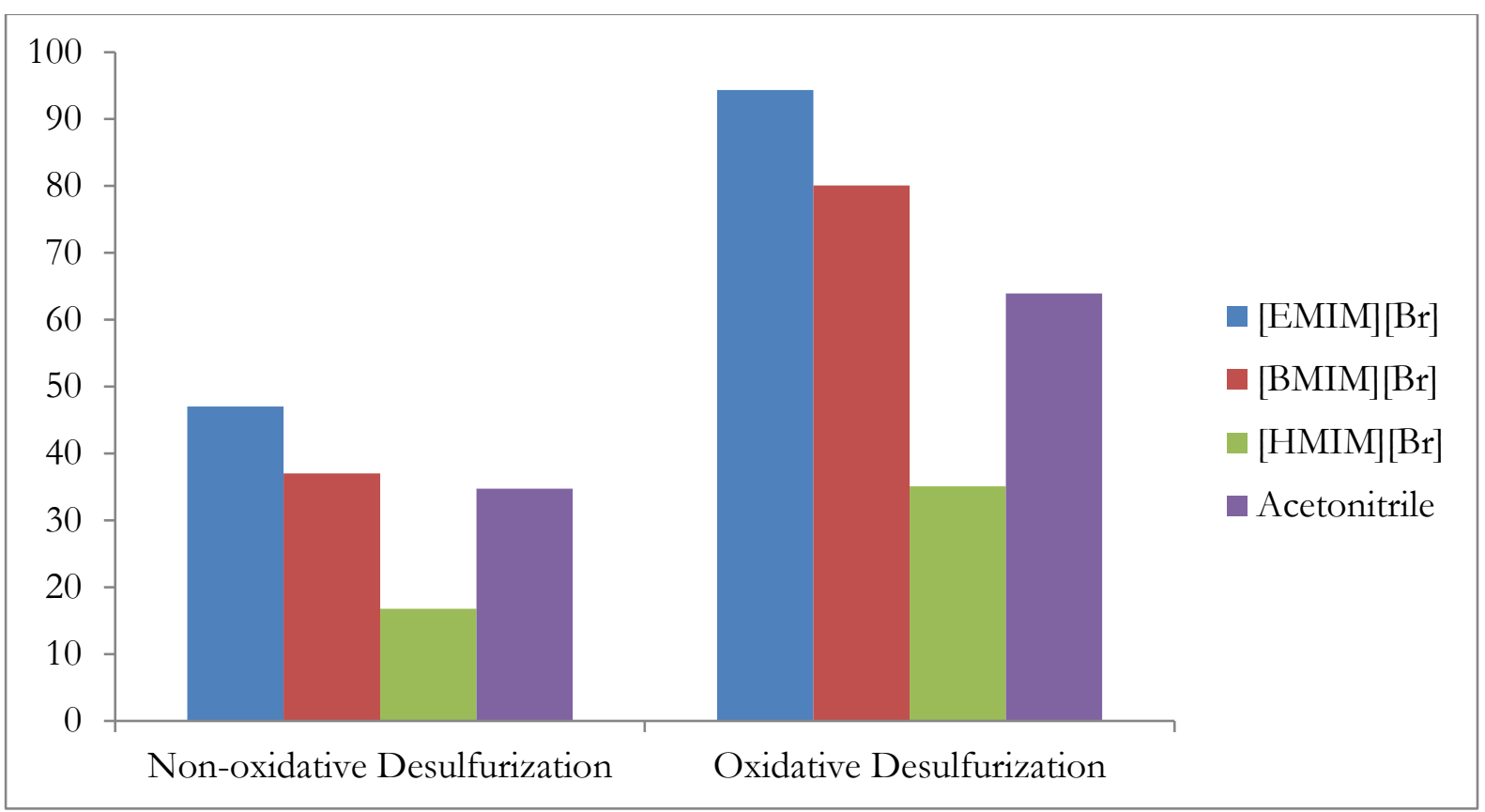

Figure 3. Percentage removal of thiophene by IL and acetonitrile in oxidative and non-oxidative desulfurization. 
Countinho, 2008) on physical properties of imidazolium-based ionic liquids show that an increase in the alkyl chain length can lead to a non-linear increase in the viscosity of the ionic liquid even at elevated pressures. An increase in alkyl chain length by 2 carbons from $[\mathrm{BMIM}][\mathrm{Br}]$ to $[\mathrm{HMIM}][\mathrm{Br}]$ increases the viscosity by two-fold at $20^{\circ} \mathrm{C}$ (Chen et al., 2006; Dehkordi et al., 2009; Lu et al., 2007; Ahosseini and Scurto, 2008; Gardas and Countinho, 2008; Pereiro et al., 2009). It is apparent that an increase in the viscosity of the IL decreases the extraction efficiency; an increase in the viscosity of the IL decreases its extent of contact with the model oil. It is worth noting that the increase in alkyl chain length decreases the polarity of the IL and this would decrease extraction efficiency for reasons of less compatible polarities between the IL and the polar sulfones and sulfoxides.

Another trend that was observed is the increase in extraction efficiency by more than two-fold upon the oxidation of thiophene into sulfoxide and sulfone. This trend was in agreement with a previous study (Lo et al., 2003; Kim et al., 2004) wherein the oxidation of thiophene and its derivatives results to a more efficient extraction of the oxidized products (sulfoxides and sulfones) into the IL phase. This increase in efficiency can be attributed to the increased polarity of sulfoxides and sulfones (Lo et al., 2003; Kim et al., 2004; Chen et al., 2006; Dekhordi et al., 2009) which better complements the polar ionic liquids used.

The recyclability (Lo et al., 2003; Kim et al., 2004; Dupont et al., 2002; Ziyauddin et al., 2009) of [EMIM][Br] for desulfurization was

Table 2. Percentage removal of thiophene in regenerated [EMIM] $[\mathrm{Br}]$ during oxidative desulfurization ${ }^{\mathrm{a}}$.

\begin{tabular}{ll}
\hline $\begin{array}{l}\text { Round of } \\
\text { Extraction }\end{array}$ & $\begin{array}{l}\text { Percentage of Sulfur } \\
\text { removed }(\%)\end{array}$ \\
\hline 1 & $94.34 \pm 0.77$ \\
2 & $93.09 \pm 0.05$ \\
3 & $92.15 \pm 0.39$ \\
\hline${ }^{a}$ Desulfurization was performed in triplicates
\end{tabular}

also assessed. After each round of desulfurization, the IL phase was washed and reused as solvent for the next desulfurization step. It was observed that [EMIM] $\mathrm{Br}]$ can be recycled three times without a significant decrease in efficiency (Table 2). This observation is in agreement with literature (Kim et al., 2004).

\section{CONCLUSION}

Oxidative desulfurization with hydrogen peroxide and acetic acid and with the ionic liquids [EMIM] Br, [BMIM] $\mathrm{Br}$, and [HMIM] $\mathrm{Br}$ as extraction solvents has been demonstrated to be an efficient method for the removal of thiophene from model oil. This method requires only moderate conditions of mixing and staging for equilibration, both performed at room temperature. The ionic liquid [EMIM] $\mathrm{Br}$ was found to be the most efficient extraction solvent among the three ionic liquids with its extraction efficiency of $94.34 \%$. It is apparent that the lenghthening of the alkyl substituent $\mathrm{R}$ on the imidazolium cation leads to a decrease in the IL's extraction efficiency. The above oxidative desulfurization method is more efficient than simple extraction with ionic liquids owing to the increased polarity of the oxidized products. Furthermore, the extraction solvent [EMIM] Br can be recycled up to three times with minimal decrease in thiophene removal efficiency.

\section{REFERENCES}

Ahosseini A, and Scurto AM. Viscosity of Imidazolium-Based Ionic Liquids at Elevated Pressures: Cation and Anion Effects. Int J Thermophys. 2008; 29:1222-1243.

Alonso L, Arce A, Francisco M and Soto A. Thiophene separation from aliphatic hydrocarbons using the 1-ethyl-3methylimidazolium ethylsulfate ionic liquid. Fluid Phase Equilibr. 2008; 270:97-102. 
Bosmann A, Datsevich L, Jess A, Lauter A, Schimtz C, and Wasserscheid P. Deep desulfurization of diesel fuel by extraction with ionic liquids. Chem Commun. 2001; (23):2494-2495.

Chen LJ, Guo SH and Zhao DS. Oxidation of Thiophenes over Silica Gel in Hydrogen Peroxide/Formic Acid System. Chinese J Chem Eng. 2006; 14(6):835-838.

Cheng DH, Chen XW, Shu Y, Wang JH. Extraction of Cytochrome C by Ionic Liquid 1-Butyl-3-trimethylsilylimidazolium

Hexafluorophosphate. Chinese J Anal Chem. 2008; 36(9):1187-1190.

Dehkordi AM, Kiaei Z and Sobati MA. Oxidative desulfurization of simulated light fuel oil and untreated kerosene. Fuel Processing Technol. 2009; 90(3):435-445.

Dupont J, de Souza RF and Suarez PA. Ionic Liquid (Molten Salt) Phase Organometallic Catalysis. Chem Rev. 2002; 102(10):36673692.

Francisco M, Arce A, and Soto A. Ionic liquids on desulfurization of fuel oils. Fluid Phase Equilibr. 2010; 294:39-48.

Fu X, Dai S and Zhang Y. Comparison of Extraction Capacities Between Ionic Liquids and Dichloromethane. Chinese J Anal Chem. 2006; 34(5):598-602.

Gardas RL and Countinho JAP. A group contribution method for viscosity estimation of ionic liquids. Fluid Phase Equilibr. 2008; 266:195-201.

Jiang $\mathrm{X}$, Nie $\mathrm{Y}, \mathrm{Li} \mathrm{C}$ and Wang $\mathrm{ZH}$. Imidazolium-based alkylphosphate ionic liquids - A potential solvent for extractive desulfurization of fuel. Fuel. 2008; 87:79-84.

Kim KS, Shin BK, and Lee H. Physical and electrochemical properties of 1-butyl-3methylimidazolium bromide, 1-butyl-3methylimidazolium iodide, and 1-butyl-3methylimidazolium tetrafluoroborate. Korean J Chem Eng. 2004; 21(5):1010-1014.
Likhanova NV, Lucero D, Flores EA, Garcia $\mathrm{P}$, Aguilar MA, Palomeque J, and Palou R. Ionic liquids screening for desulfurization of natural gasoline by liquid-liquid extraction. Molecular Divers. 2010; 14(4):777-87.

Lo WH, Yang HY, and Wei GT. One-pot desulfurization of light oils by chemical oxidation and solvent extraction with room temperature ionic liquids. Green Chem. 2003; 5:639-642.

Lu L, Cheng S, Gao J, Gao G and He M. Deep Oxidative Desulfurization of Fuels Catalyzed by Ionic Liquid in the Presence of $\mathrm{H}_{2} \mathrm{O}_{2}$. Energy Fuels. 2007; 21:383-384.

Pereiro AB, Veiga HM, Esperanca JS and Rodriguez A. Effect of temperature on the physical properties of two ionic liquids. J Chem Thermodyn 2009; 41(12):1419-1423.

Seeberger A and Jess A. Desulfurization of diesel oil by selective oxidation and extraction of sulfur compounds by ionic liquids-a contribution to a competitive process design. Green Chem. 2009; 12:602-608.

Zhao DS, Sun ZM, Li FT and Shan HD. Optimization of oxidative desulfurization of dibenzothiophene using acidic ionic liquid as catalytic solvent. Journal of Fuel Chemistry and Technology. 2010; 37(2):194-198.

Zhao DS, Wang YN, Duan EH and Zhang J. Oxidation desulfurization of fuel using pyridinium-based ionic liquids as phasetransfer catalysts. Fuel Processing Technol. 2009; 91:1803-1806.

Ziyauddin S, Deshmukh KM, Bhor MD and Bhanage BM. Bronsted acidic ionic liquid as an efficient and reusable catalyst for transesterification of $\beta$-ketoesters. Catal Commun. 2009; 10(6):833-837. 
Harold Henrison C. Chiu, Susan D. Arco, Zhang Chun Ping, and Nelson R. Villarante 